\title{
Design and Optimization of Inductive Power Transmission for Implantable Sensor System
}

\author{
Enver G. Kilinc, Catherine Dehollain \\ RFIC-Group \\ Ecole Polytechnique Fédérale de Lausanne \\ Lausanne, Switzerland \\ enver.kilinc@epfl.ch, catherine.dehollain@epfl.ch
}

\author{
Franco Maloberti \\ Integrated Microsystem Laboratory \\ Università degli Studi di Pavia \\ Pavia, Italy \\ franco.maloberti@unipv.it
}

\begin{abstract}
This paper presents a methodology to design and to optimize inductive power link for biomedical applications. The importance of the operation frequency on the application is expressed. A model of inductive link is presented. The dimensions of the coils are compatible with the size of a mouse and the mouse cage. The simulation results are in good agreement with the analysis.
\end{abstract}

\section{INTRODUCTION}

The advances in microelectronic technologies allow fabricating very small circuits that can be implantable as a sensor system for a body. These sensor systems help monitoring patients online. The response of the body to the medicine is recorded, so that, the patient can be treated personally due to its metabolism. Another important issue is investigating the side-effects of the used anti-inflammatory drugs to treat patients individually. The monitoring system measures the toxicity of the drugs, the drug level, ATP (Adenosine Triphosphate), glucose level, temperature, and $\mathrm{pH}$ level.

The developed biochip system will be implanted to a laboratory mouse, which can be used for biomedical and genetic researches and also for investigating new treatments [1]. Fig. 1 shows the miniature concept of a typical implantable biochip system.

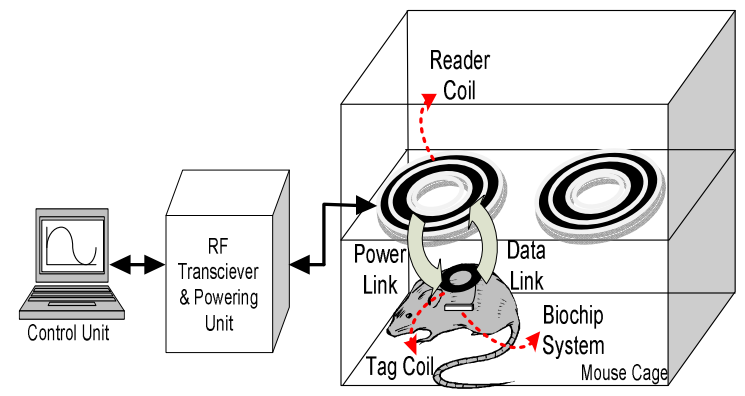

Figure 1. Miniature concept of a typical implantable biochip system
One solution is to use the cables for data transmission and powering the sensor system. However, these cables are limits the mobility of the subject animal and may also cause infections. An alternative solution is to use batteries on the tag side. By this way, a fully implantable system can be obtained. However, many surgeries may be necessary to replace the batteries at the end of their life time. Additionally, the batteries increase the total weight of the system and also create toxic effect for the subject. To solve this, wireless power transmission is the most appropriate solution in order to get an infinite life time with respect to remote powering. There are different ways to transmit wireless power such as ultrasound, magnetic and electromagnetic [1-3]. Acoustic waves propagate in media such as water; however, they are strongly attenuated in air. Henceforth, for this application, the magnetic and electromagnetic methods are compared in this study.

The boundary between magnetic coupling and electromagnetic power harvesting is defined by $d=\lambda / 2 \pi$, where, $d$ and $\lambda$ are the distance and the wavelength of the signal, respectively. For a chosen wavelength, if the distance between the coils is smaller than $d$, the magnetic coupling gives more efficient wireless power transmission [2].

In the next section, the selection of inductive link frequency is discussed. In Section III, the design of inductive power link is summarized including analysis for increasing the efficiency. Section IV compares the hand calculations and simulation results. Conclusions are given in Section V.

\section{FREQUENCY SELECTION}

The frequency is one of the most important parameters for efficient power transmission in the system. For an inductive link in the air, the induced voltage at the transponder side is given by [4]:

$$
V_{I N D}=\mu_{0} \cdot A \cdot N \cdot \omega \cdot H_{\text {eff }}
$$

where, $\mu_{0}, \mathrm{~A}, \mathrm{~N}, \omega$, and $\mathrm{H}_{\text {eff }}$ are the permeability constant of air, the loop area of the tag coil, the number of turns of the tag coils, the angular frequency of the magnetic coupling, and the 
effective field strength of the magnetic field. The higher the frequency, the higher induced voltage is. In addition, higher operation frequencies allow higher data rates due to higher bandwidth. However, in the case of biomedical applications, the absorption of the body tissues increases with frequency [5]. Moreover, the parasitic effects of the inductance such as skin effect, eddy currents, etc. increase with frequency [6]. By considering all the aforementioned issues, the frequency is chosen as $13.56 \mathrm{MHz}$ which is one of the inductive application band determined by regulations [7]. Moreover, it allows maximum magnetic field strength, higher data bandwidth, and less interference compared to other options, due to the standards dedicated to this band [4].

\section{INDUCTIVE POWER LINK AND EFFICIENCY ANALYSIS}

In magnetic coupling, the reader and tag coils must be designed carefully because there are many parameters which affect the power efficiency in an inductive link. Furthermore, these parameters are application dependent which means that the inductive link must be designed and optimized for each application. The distance between the coils is one of the most important parameters to have efficient power transmission, since the power transmission efficiency is strongly proportional to inverse of the distance $\left(1 / \mathrm{d}^{6}\right)$ in magnetic coupling [2]. Hence, the distance between the reader coil and the tag coil should be minimized. Additionally, the coil geometry has a significant effect on power efficiency [8]. For a defined boundary box, the square spiral coil is preferable to get maximized power transfer area. The additional dead-zones for the circular spiral coil are displayed as red in Fig. 2.
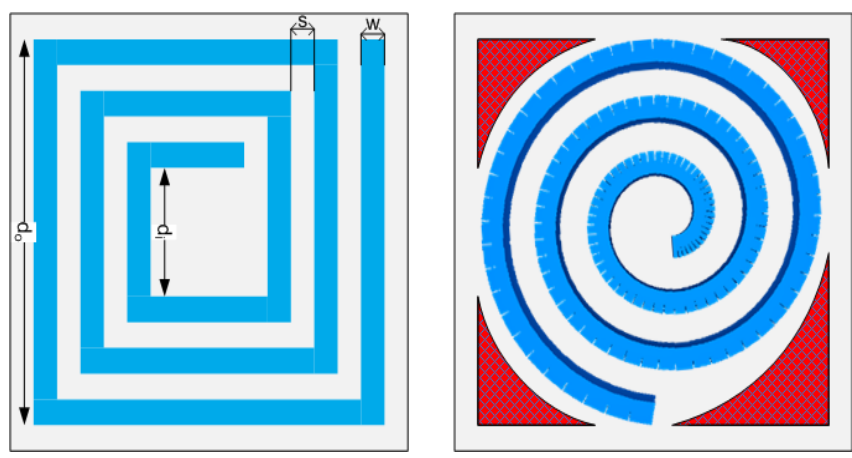

Figure 2. Square and circular shaped coils

The efficiency of the power transmission is mostly related to inductive link efficiency. Fig. 3 shows the simplified model of the inductive power link. In this figure, $L_{i}, R_{i}$, and $C_{p i}$ represent the self inductance, series resistance, and parasitic capacitance, respectively. In addition, the mutual inductance and load resistance are represented as $M$ and $R_{\text {Load }}$, respectively. $C 1$ and $C 2$ are the capacitances to tune the link at resonance frequency.

\section{A. Modeling of Inductive Link}

The magnetic field is formed by current flowing through the reader coil and this magnetic field induces current flowing through tag coil. The value of the induced current is related to the inductance values of $L_{1}$ and $L_{2}$. The inductance value of the square spiral coil in Fig. 2 can be calculated as [9]:

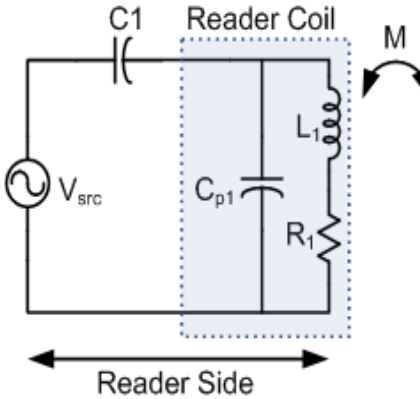

Reader Side

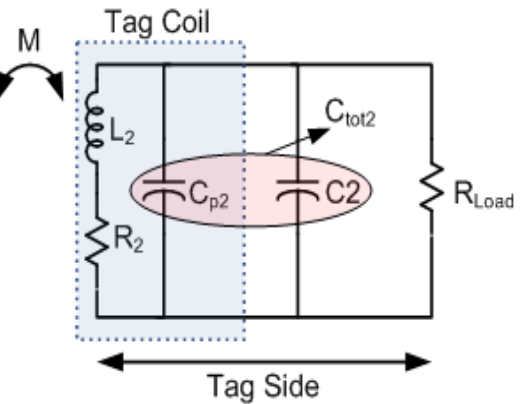

Tag Side
Figure 3. Simplified model of the inductive power link

$$
L=\frac{1.27 \cdot \mu_{0} n^{2} d_{\text {avg }}}{2}\left[\ln \left(\frac{2.07}{\rho}\right)+0.18 \rho+0.13 \rho^{2}\right]
$$

where, $n$ is the number of turns of the spiral coil. $d_{i}$ and $d_{o}$ are the inner and the outer diameter of the coil. $d_{\text {avg }}$ is the average diameter $\left(\left(d_{i}+d_{o}\right) / 2\right) . \rho$ is the fill ratio and defined as $\rho=\left(d_{o}-d_{i}\right) /\left(d_{i}+d_{o}\right)$.

Quality factor of the inductor is also another important parameter that affects the power efficiency, and is related to the parasitic resistance and capacitance of the inductor. The parasitic resistance also increases with frequency due to the skin and proximity effects. By considering skin effect, the resistance can be calculated as [10]:

$$
R_{a c}=R_{d c} \frac{t}{\delta \cdot\left(1-e^{-t / \delta}\right)}, \delta=\sqrt{\frac{\rho_{c}}{\pi \cdot \mu \cdot f}}, R_{d c}=\rho_{c} \frac{l}{w \cdot t}
$$

where, $t, w$, and $\rho_{c}$ are thickness, width, and resistivity of the conductor, respectively. $l, f$, and $\delta$, are the total length of the conductor line, operation frequency, and the skin depth, respectively. $\mu$ is the permeability constant and defined as $\mu=\mu_{0} \mu_{r}$ where $\mu_{r}$ is relative permeability of the conductor.

Finally, the parasitic capacitance is required to model the coil and to define the self-resonance frequency in order to choose optimum inductance. The capacitance is defined by [11]:

$$
C_{p}=\left(\alpha \varepsilon_{r c}+\beta \varepsilon_{r s}\right) \varepsilon_{0} \frac{t}{s} l
$$

where, $t, s, l$, and $\varepsilon_{0}$ are the thickness of the conductor, the spacing between two conductors, the total length of the conductor line, and the dielectric constant of the air, respectively. The total capacitance is the sum of the coating capacitance and substrate capacitance; hence, $\varepsilon_{r c}$ and $\varepsilon_{r s}$ are the relative dielectric constants of coating and substrate materials. Moreover, $(\alpha, \beta)$ is assumed as $(0.9,0.1)$ in the case of air and printed circuit board substrate (FR4) [11].

The mutual inductance is the key parameter in the link efficiency. Assuming a perfect alignment, the total mutual inductance between the reader and the tag coils is expressed by [12]: 


$$
M_{12}=\theta \sum_{i=1}^{N_{1}} \sum_{j=1}^{N_{2}} M\left(x_{i}, y_{j}, d_{12}\right)
$$

where, $\theta$ is a constant and changes with the shape of the coil. It is found empirically as 1.3 for square-shaped coils [11]. Additionally, $N_{l}, N_{2}, x_{i}, y_{j}$ and $d_{12}$ are the number of turns in reader coil, the number of turns in tag coil, the radius of $i^{\text {th }}$ turn in reader coil, the radius of $\mathrm{j}^{\text {th }}$ turn in tag coil and distance between coils, respectively. $M\left(x, y, d_{12}\right)$ is defined as [12]:

$$
M\left(x, y, d_{12}\right)=\mu_{0} \sqrt{x \cdot y}\left[\left(\frac{2}{\gamma}-\gamma\right) K(\gamma)-\frac{2}{\gamma} E(\gamma)\right]
$$

where, $\gamma$ is

$$
\gamma=\sqrt{\frac{4 x y}{(x+y)^{2}+d_{12}^{2}}} .
$$

\section{B. Link Power Efficiency}

The efficiency is maximized when LC-tanks are tuned at the working frequency $(\omega)$. In an inductive power link, $L_{l}$ and $L_{2}$ coils are tuned with $C_{t o t l}$ and $C_{\text {tot } 2}$, respectively. Assuming $\omega=\omega_{01}=\omega_{02}$, the efficiency of the transmitted power from source to load is dominated by receiver side efficiency $\left(\eta_{1}\right)$ and tag side efficiency $\left(\eta_{2}\right)$ and can be expressed by [13]:

$$
\eta_{12}=\eta_{1} \cdot \eta_{2}=\frac{X \frac{R_{2}}{R_{\text {Load }}}}{\left(1+\frac{R_{2}}{R_{\text {Load }}}+X \frac{R_{2}}{R_{\text {Load }}}\right)\left(1+\frac{R_{2}}{R_{\text {Load }}}\right)} .
$$

where, $X$ is $M^{2} \cdot \omega^{2} / R_{1} R_{2}$ or $k^{2} Q_{1} Q_{2} . k$ is the coupling coefficient and, $Q_{1}$ and $Q_{2}$ are the quality factors of unloaded reader coil and unloaded tag coil, respectively. Quality factor $(Q)$ is defined as $Q=\omega L / R_{a c}$ for low frequencies. The optimal $R_{\text {Load }}$ can be found by [13]:

$$
R_{\text {Load }}=R_{2} \sqrt{1+X}
$$

By taking $R_{\text {Load }}$ as optimum, the maximum efficiency can be calculated as:

$$
\eta_{12}=\frac{k^{2} Q_{1} Q_{2}}{\left(1+\sqrt{1+k^{2} Q_{1} Q_{2}}\right)^{2}} .
$$

The maximum power efficiency can be obtained by maximizing not only the coupling factor, but also the quality factors of the coils. Hence the coils must be designed carefully to get higher coupling factor as well as higher quality factors.

\section{Misalignment Analysis}

The previous equations are done by taking into account the perfect alignment case. However, there are two important misalignment cases:

1) Angular Misalignment: In angular misalignment case, the tag coil is turned by an angle $\alpha$ and the centers of the coils are aligned.
2) Lateral Misalignment: In lateral misalignment case, the tag coil is moved by a distance $\Delta$ and the coils are situated in parallel planes.

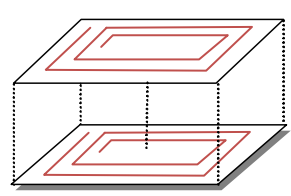

a)

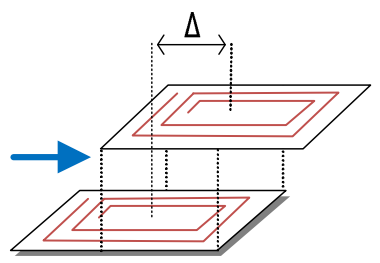

c)

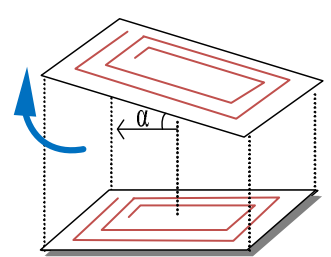

b)

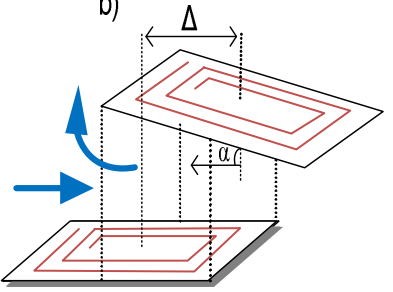

d)
Figure 4. a) Perfect alignment case, b) Angular misalignment case, c) Lateral misalignment case, d) Both angular and lateral misalignment case

Fig. 4 represents perfect alignment, angular misalignment, lateral misalignment, and both angular and lateral misalignment cases of the reader and tag coils. The link power efficiency will be reduced by both angular and lateral misalignment cases. Hence, these cases should be considered during the coil design.

In the angular misalignment case, the magnetic field is changing with respect to $\cos \alpha$ [14]. When the tag coil is parallel to the reader coil $(\alpha=0)$, the magnetic field is maximized. However, the magnetic field is minimized when the tag coil is situated perpendicular to the reader coil $(\alpha=90)$.

In the lateral misalignment case, the magnetic field is related to displacement $\Delta$ [14]. When the displacement $\Delta$ is increased, the link efficiency decreases.

\section{Case Study: Remote Powering of a Mouse IMPLANT FOR BIOCHIP SYSTEMS}

To find the optimum inductive power link, the coils are designed in terms of geometrical parameters. In order to do this, a MATLAB code is developed to find the optimum inductive link by using the expressions presented in Section III. Furthermore, the results are verified and tuned by using HFSS program.

Some parameters are limited due to the application such as the distance $\left(d_{12}\right)$ between the coils, the outer diameter of tag coil $\left(d_{o 2}\right)$. Other parameters have a degree of freedom. They should be chosen carefully to get optimum inductive power link. Table I presents the fixed parameters for this application.

The optimal value of the outer diameter of the reader coil $\left(d_{o l}\right)$ which maximizes the magnetic strength is defined by [4]:

$$
d_{o 1}=d_{12} \cdot 2 \sqrt{2} \text {. }
$$




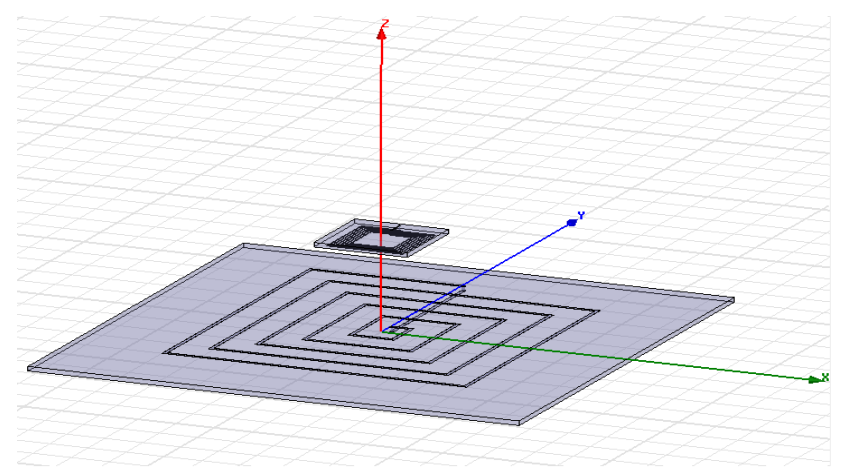

Figure 5. 3D model of the inductive power link on HFSS program

TABLE I.

DESIGN PARAMETERS LIMITED BY APPLICATION

\begin{tabular}{|c|c|}
\hline Parameter & Value \\
\hline Link operation frequency $(\mathrm{f})$ & $13.56 \mathrm{MHz}$ \\
\hline Distance between coils $\left(\mathrm{d}_{12}\right)$ & $30 \mathrm{~mm}$ \\
\hline Tag coil outer diameter $\left(\mathrm{d}_{\mathrm{o} 2}\right)$ & $20 \mathrm{~mm}$ \\
\hline Minimum spacing between conductors ${ }^{\mathrm{a}}(\mathrm{s})$ & $150 \mu \mathrm{m}$ \\
\hline Minimum width of conductor ${ }^{\mathrm{a}}(\mathrm{w})$ & $150 \mu \mathrm{m}$ \\
\hline
\end{tabular}

By placing the coils under the cage to minimize the distance $\left(d_{12}=3 \mathrm{~cm}\right)$, the optimal outer diameter $\left(d_{o l}\right)$ can be derived as $8.48 \mathrm{~cm}$. With respect to power efficiency, the optimal $d_{o 1}$ is calculated as $9.5 \mathrm{~cm}$ as shown in Fig. 6 .

In the project, the sensor system is implanted inside a laboratory mouse which can move in the cage and the tag coil position will be changed compared to the reader coil. In conclusion, the lateral and also angular misalignment problems can be occurred. The angular misalignment may not be significant issue under assumption of the mouse is moving parallel to the basement of the cage most of the time. However, the lateral misalignment will be more hazardous for link efficiency. Increasing $d_{o l}$ too much may not be an optimum solution, because the lateral misalignment is also increasing which decreases the link efficiency. Hence, $d_{o l}$ is chosen as $8 \mathrm{~cm}$ due to the cage geometry which is depicted in Fig. 7 and also minimizing the misalignment problem. However, to cover all the basement of the cage, there should be an array of coils. On the other hand, powering all of the coils at the same time creates interferences to each other and also increases the total power consumption. To solve this, a magnet is placed on the tag side and a sensor is added each of the reader coils [15]. When the mouse comes on the coil, the magnet is detected and the coil is powered.

There are still several other parameters which affect the power efficiency. For a chosen conductor width, the selfinductance value increases with increasing the number of turns but the self-resonance frequency decreases. Moreover, the quality factor increases proportional to conductor width. However, the parasitic capacitances increase, and therefore, the self-resonance frequency decreases. Hence, the optimum reader and tag coil design must be investigated to get maximum link efficiency in terms of coil geometry parameters.

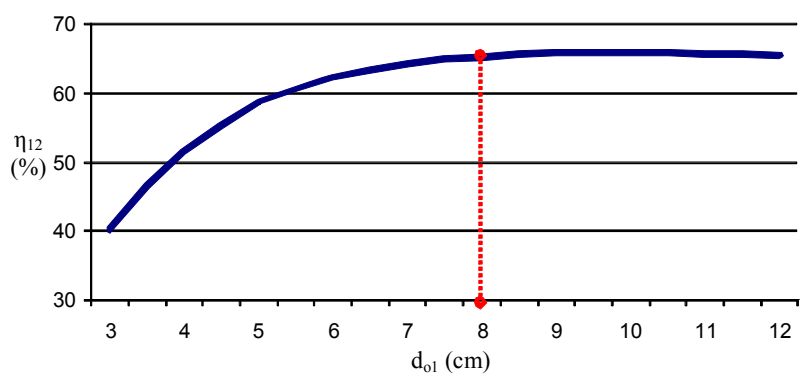

Figure 6. Efficiency $\left(\eta_{12}\right)$ versus outer diameter of the reader coil $\left(\mathrm{d}_{\mathrm{o} 1}\right)$

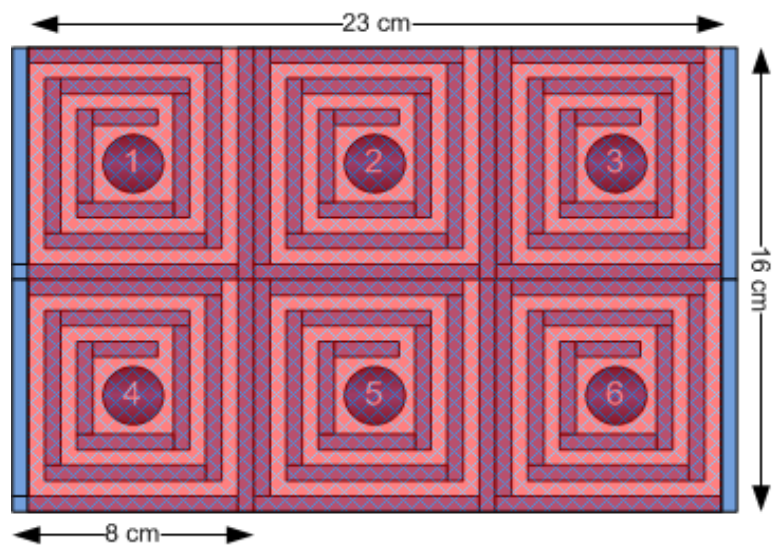

Figure 7. The array of coils under basement of the cage

Due to the previous equations, the link power efficiency is related to not only coupling coefficient $(k)$, but also quality factors of the coils $\left(Q_{1}, Q_{2}\right)$. Hence, the inductive link geometry is optimized in MATLAB using the aforementioned equations considering $k, Q_{1}, Q_{2}$ together by changing the coil parameters such as number of turns $\left(\mathrm{n}_{1}, \mathrm{n}_{2}\right)$, conductor width $\left(\mathrm{w}_{1}, \mathrm{w}_{2}\right)$ and, spacing between conductors $\left(\mathrm{s}_{1}, \mathrm{~s}_{2}\right)$. The results of the optimization are summarized in Table II. Additionally, the optimized coils are simulated in HFSS. The comparison between the analysis and simulation are shown in Table III.

\section{CONCLUSION}

In this paper, the differences between different power harvesting methods are discussed. The magnetic coupling is more efficient with respect to electromagnetic power harvesting, in terms of transmitted power due to the short distance between the coils. Moreover, the importance of the operation frequency is emphasized. Additionally, an inductive link is presented and analyzed in terms of power efficiency. Besides, the lateral and angular misalignment conditions are focused. A method of geometric optimization for inductive links is described. Finally, a case study for remote powering of a mouse implant for biochip systems using inductive power link is presented.

For the future work, the array of reader coils and tag coil will be fabricated including the magnet and the sensors and will be characterized comparing with analysis and simulation. In addition, a control loop mechanism will be investigated to minimize lateral misalignment issue. 
TABLE II.

OptiMAL INDUCTIVE LINK COIL DESIGNS

\begin{tabular}{|c|c|c|}
\hline Parameter & Reader Coil & Tag Coil \\
\hline Outer Diameter $\left(\mathrm{d}_{\mathrm{o}}\right)$ & $8 \mathrm{~cm}$ & $2 \mathrm{~cm}$ \\
\hline Inner Diameter $\left(\mathrm{d}_{\mathrm{i}}\right)$ & $10 \mathrm{~mm}$ & $11 \mathrm{~mm}$ \\
\hline Number of turns $(\mathrm{n})$ & 5 & 6 \\
\hline Width of conductor $(\mathrm{w})$ & $1 \mathrm{~mm}$ & $250 \mu \mathrm{m}$ \\
\hline Spacing between conductors $(\mathrm{s})$ & $7.5 \mathrm{~mm}$ & $600 \mu \mathrm{m}$ \\
\hline
\end{tabular}

TABLE III.

COMPARISON BETWEEN ANALYSIS AND HFSS SIMULATION RESULTS

\begin{tabular}{|c|c|c|}
\hline Parameter & Analysis & Simulation \\
\hline Reader Coil Inductance $\left(\mathrm{L}_{1}\right)$ & $1.075 \mu \mathrm{H}$ & $1.002 \mu \mathrm{H}$ \\
\hline Tag Coil Inductance $\left(\mathrm{L}_{2}\right)$ & $0.9 \mu \mathrm{H}$ & $0.75 \mu \mathrm{H}$ \\
\hline Reader Coil Resistance $\left(\mathrm{R}_{1}\right)$ & $0.597 \Omega$ & $0.56 \Omega$ \\
\hline Tag Coil Resistance $\left(\mathrm{R}_{2}\right)$ & $0.715 \Omega$ & $0.74 \Omega$ \\
\hline Reader Coil Quality Factor $\left(\mathrm{Q}_{1}\right)$ & 152 & 156 \\
\hline Tag Coil Quality Factor $\left(\mathrm{Q}_{2}\right)$ & 81 & 85 \\
\hline Mutual Inductance $\left(\mathrm{M}_{12}\right)$ & $36.04 \mathrm{nH}$ & $37.12 \mathrm{nH}$ \\
\hline Power Efficiency $(\eta 12)$ & $65.5 \%$ & $66.7 \%$ \\
\hline
\end{tabular}

\section{ACKNOWLEDGMENT}

The authors are grateful to Prof. J. R. Mosig from LEMA Laboratory, EPFL and his group for their support of the HFSS simulation tool. This project is supported by Swiss National Foundation (SNF) through Sinergia Initiative.

\section{REFERENCES}

[1] P. Cong et. al., "Novel long-term implantable blood pressure monitoring system with reduced baseline drift," Proc. IEEE EMBC '06, pp. 1854-1857, 2006.

[2] D. C. Yate et. al., "Optimal transmission frequency for ultralow-power short-range radio links," IEEE Trans. Circuits Syst. I, vol. 51, no. 7, pp. 1405-1413, July 2004.

[3] F. Mazzilli et. al., "In-vitro platform to study ultrasound as source for wireless energy transfer and communication for implanted medical devices," to be presented in Proc. IEEE EMBC '10.

[4] K. Finkenzeller, "RFID Handbook," John Wiley and Sons Ltd, 1999.

[5] P. Vaillantcourt et. al., "EM radiation behaviour upon biological tissues in a radio-frequency power transfer link for a cortical visual implant," Proc. IEEE EMBC'97, pp. 2499-2502, 1997.

[6] S. Mei and Y. I. Ismail, "Modeling skin and proximity effect with the reduced realizable RL circuits", IEEE Trans. VLSI Syst., vol. 12, no. 4, pp. 437-447, 2004.

[7] ERC-REC 70-03, "Inductive Applications," in Annex 9, Oct 2009.

[8] K. M. Silay et. al., "Improvement of power efficiency of inductive links for implantable devices," Proc. PRIME'08 Conf., 2008, pp. 229-232.

[9] S. S. Mohan et. al., "Simple accurate expressions for planar spiral inductances," IEEE J. Solid-State Circ., vol. 34, pp. 1419-1424, Oct. 1999.

[10] O. Atasoy and C. Dehollain, "A study for remote powering of a knee prosthesis through inductive link," to be presented in Proc. PRIME'10 Conf., 2010.

[11] U.- M. Jow, and M. Ghovanloo, " Modeling and optimization of printed spiral coils in air, saline, and muscle tissue environments," IEEE Trans. Biomed. Circuits Syst., vol. 3, pp. 339-347, 2009.

[12] C. M. Zierhofer and E. S. Hochmair, "Geometric approach for coupling enhancement of magnetically coupled coils," IEEE Trans. Biomed. Eng., vol. 43, pp. 708-714, July 1996.

[13] B. Lenaerts and R. Puers, "Omnidirectional Inductive Powering for Biomedical Implants," Springer, 2009.

[14] K. Fotopoulou and B. W. Flynn, "Wireless powering of implanted sensors using RF inductive coupling," IEEE Sensors 2006 EXCO, Korea, Oct 2006.

[15] H. G. Lim et. al., "A method for reducing body exposure to electromagnetic field of pillow type wireless charger in fully implantable middle ear hearing device," IEICE Electronics Express, vol. 6 , no. 18 pp.1318-1324, 2009. 